Check for updates

Cite this: RSC Adv., 2019, 9, 41993

\title{
Harnessing platelets as functional vectors for contrast enhanced ultrasound imaging and fluorescence imaging
}

\author{
Chuang Gao, $\dagger^{a}$ Pravin Bhattarai, (ID) $\dagger^{a}$ Yiming Zhou, ${ }^{a}$ Nisi Zhang, ${ }^{a}$ Sadaf Hameed, ${ }^{a}$ \\ Xiuli Yue*b and Bo Zhao*bc
}

The recent progress in the development of highly biocompatible nanoplatforms mostly encompasses the use of biological excipients such as red blood cells, cancer cell membranes, and also platelets. Such specialized vectors, if mimicked correctly, have intrinsic ability to navigate through the biological system and perform their intended action without eliciting any cascade of inflammatory processes. Naturally, platelets have been found to accumulate in the wound sites and also interact with circulating tumor cells (CTCs). Inspired by the targeting ability of platelets and the clinical success of ultrasound, herein we developed a novel ultrasound contrast agent (UCA) by backfilling of an insoluble gas into the platelets after lyophilization ex vivo. The as-prepared platelet-based ultrasound contrast agent (P-UCA) disguised the structural integrity of the natural platelets with an average diameter of $3.1 \pm 0.4 \mu \mathrm{m}$, and could enhance the ultrasound signal both in vitro and in vivo. Besides, we further evaluated that such platelet particles could facilitate active loading of ICG molecules for prolonged in vivo fluorescence imaging compared to the free ICG. Taking all the results together, we established that biological structures such as platelets could be repurposed ex vivo as a"shell" to encapsulate gas and be further extended to load ICG for real-time ultrasound and fluorescence imaging respectively. This not only indicates many potential uses of these MBs in the diagnosis of platelet-related diseases, such as vascular damage, thrombosis, and atherosclerosis, but also serves as a powerful platform with multimodal theranostic capability after active loading of a variety of therapeutic and diagnostic agents.

Received 5th July 2019

Accepted 23rd November 2019

DOI: 10.1039/c9ra05118j

rsc.li/rsc-advances increasing role of platelets in the progression of cancer and spread of tumor cells, also called as distant metastases, by interacting directly with the circulating tumor cells (CTCs) in the bloodstream or aggregating on the surface of the tumor cells. $^{4-6}$ These unique biological function of platelets provide new opportunities in the biomedical field and have inspired researchers to envision platelet-mimicking biomimetic delivery systems to unravel poorly understood or biologically complex applications. ${ }^{7,8}$ However, the conventional cell membrane modification strategies are still insufficient to harness the complete functional structure of naive circulating platelets in either way: (i) the modified nanostructure fails to mimic the complex biochemical interactions that usually occurs when natural platelets interacts with and binds to the endothelium, (ii) membrane-cloaked synthetic polymeric or liposomal nanostructures fails to mimic the unique discoid shape or distensibility of natural platelets that usually dictates the natural transportation mechanisms such as hemodynamics or endothelial margination for effective targeting at injury-sites. On the other hand, despite the huge advantages of biological membrane-derived delivery systems, only a very little attention has been paid in the diagnostic field via real-time image tracking system in vivo. The clinical relevance of designing 
excellent imaging probe is noteworthy to diagnose and monitor physiological and pathological information of diseases in vivo, guide surgical interventions, and evaluate therapeutic efficacy and prognosis ${ }^{9}$. For instance, Dai et al. repurposed human platelets by loading a kabiramide (KabC) drug for minimizing platelet aggregation and further entrapped small molecular imaging probes (Cy5/Cy7) for fluorescence imaging. ${ }^{10}$ Such repurposed platelets could easily target tumor cells and allow high contrast fluorescence imaging of tumors in vivo. Nevertheless, other imaging modalities such as US, CT, MRI, PET, and SPECT can equally benefit clinicians for excellent diagnosis and prognosis of tumors. However, owing to high sensitivity, robustness, relative inexpensiveness, and adequate safety mainly due to nonionizing radiations, clinicians rely more on US and NIRF imaging.

An ultrasound contrast agent (UCA) has emerged as a powerful platform for clinical diagnosis of blood disease and tumor. Contrast-enhanced ultrasound imaging is widely applied for general tissue delineation and perfusion by changing the acoustic impedance of the tissue. Indeed, several microbubbles (MBs) such as SonoVue, Sonozoid, and Definity, have already been approved by FDA for cancer diagnosis mainly due to their significantly enhanced echogenic signals. The most routinely used UCAs are prepared as an aqueous dispersion of gas (e.g. perfluorocarbon) MBs coated with a thin shell. Despite the great success of UCA, the use of nerd shell materials, without any functional significance, limits the biological ability, for example, tissue targeting ability and microenvironment signal detection. Therefore, there is a pressing need to develop a new class of functional UCA that has inherent targeting ability and high signal to noise ratio (SNR) in vivo.

Similarly, fluorescence imaging in the near-infrared region can facilitate ultrasensitive detection at higher penetration depth non-invasively. Over the past several years, a plethora of fluorophores that can absorb and emit light at higher wavelengths have been increasingly used in biomedical applications. Notably, indocyanine green (ICG, Abs - 740 nm, Em - 800 nm), the only NIR organic dye approved by the U.S. Food and Drug Administration (FDA) for human medical imaging and diagnosis in the clinics, ${ }^{\mathbf{1 1}}$ is the best possible candidate for NIRF imaging till date. ${ }^{\mathbf{1 2} 13}$ However, there are several major challenges for using ICG in long-term tracking applications, such as a tendency to aggregate, photostability, photobleaching, ${ }^{14}$ rapid clearance from the body with a short half-life of 2-4 min. ${ }^{12,15}$ Thus, ICG cannot be utilized for sensitive and prolonged imaging in vivo. ${ }^{16}$ In order to improve this, cell membranederived functional materials having intact biological function have attracted profound attention. Recently, a plethora of nanoparticle is developed and introduced to address the intrinsic issues of ICG degradation, rapid blood clearance and in vivo imaging. Taking this into account, we envisioned and constructed a novel ultrasound contrast agent by filling the naïve platelets with $\mathrm{C}_{3} \mathrm{~F}_{8}$ gas (named as platelet $\mathrm{MBs} / \mathrm{P}-\mathrm{UCA}$ ) that can navigate through tissues, anchor to the diseased site and generate strong contrast when exposed to the ultrasonic irradiation (Scheme 1). Additionally, we further demonstrated the active loading of ICG molecules in the MBs and observed prolonged fluorescence imaging in vivo. The biologically active protective layer greatly helped ICG to retain its intrinsic fluorescence ability in the physiological milieu and enabled specific accumulation at the targeted site. To the best of our knowledge, this proof-of-concept, first time to construct a UCA derived from a biologically active component with an intact physiological function, should unequivocally proclaim its importance in the area of biomedical imaging demanding high contrast, better resolution and real-time monitoring in vivo.

\section{Results and discussion}

\subsection{Preparation and characterization of P-UCA}

P-UCA was easily prepared by backfilling the perfluoropropane into the lyophilized platelet under the protection of trehalose. For the characterization of the P-UCA, PBS was injected into the vial to rehydrate the P-UCA, followed by gently shaking of the vial to fill the gas into the P-UCA. The morphology of P-UCA was studied by using optical microscopy with fresh platelets as a control. As shown in Fig. 1, rehydrated platelets showed a similar shape compared with the fresh platelets, which indicated that during the freeze-drying and rehydration process the structure of platelet was not damaged due to the protection of trehalose. The quantitative results show that about $80 \%$ of platelets can be successfully recovered from lyophilization and without any rupture. The volume distribution results showed that the platelet size slightly increased from $2.7 \pm 0.5 \mu \mathrm{m}$ (fresh platelet) to $3.1 \pm 0.4 \mu \mathrm{m}$ (P-UCA), probably because the $\mathrm{C}_{3} \mathrm{~F}_{8}$ gas could not be discharged from the inside of the platelets during the rehydration process, resulting in a slight expansion of the platelets.

To confirm whether the gas was filled into the platelets, we used gas chromatograph-mass spectrometer (GC-MS) to analyze the gas content inside the platelet MBs and compared it with the commercially available microbubble, Sonozoid (Fig. 2). The rehydrated P-UCA was injected into the airbag and treated with the ultrasonic water bath overnight, then the gas sample was analyzed by GC-MS. The results indicated that the P-UCA contained $\sim 1.3 \%$ of the gas in the commercial lipid MB when the concentration of the MBs was same. It implied the potential ultrasound imaging capability of the P-UCA. In brief, these results are reasonably good to justify that P-UCA having excellent structural integrity and good stability is likely a potential

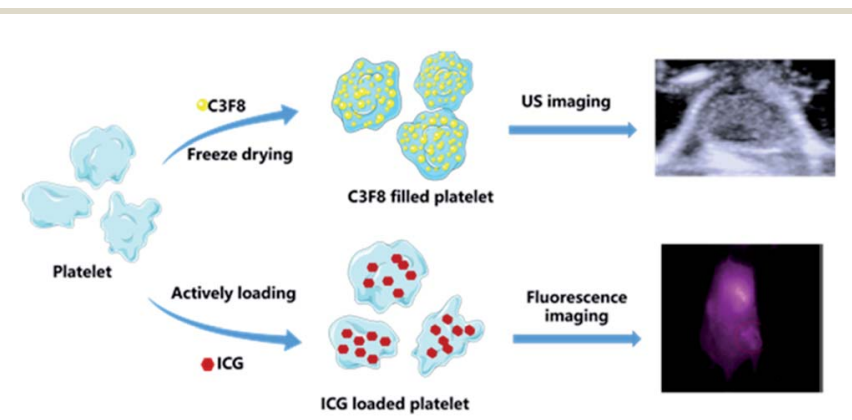

Scheme 1 Schematic illustration of platelet loading $\mathrm{C}_{3} \mathrm{~F}_{8}$ gas and ICG for tumor ultrasound and fluorescence imaging. 


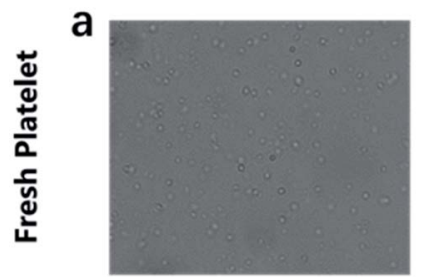

b
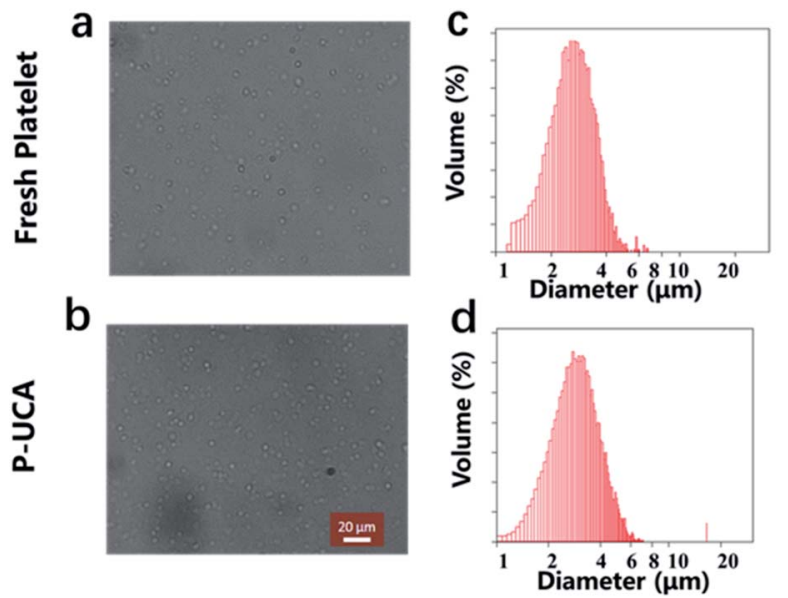

Fig. 1 Optical microscopy image of a suspension of (a) fresh platelet and (b) P-UCA; size distribution of (c) fresh platelet and (d) P-UCA measured by Coulter Multisizer III.

candidate for the further study of imaging and biological applications.

\subsection{Ultrasound echogenicity of P-UCA}

Herein, we first investigated the echogenic ability of P-UCA in vitro using a simple latex tube setup. The suspension of P-UCA was prepared in PBS solution and injected into the circulating saline filled latex tube simulating the blood vessel. For this, four groups were separated as PBS (negative control), freshly isolated platelets, freeze drying medium and platelet MBs. As shown in Fig. 3a, we observed no US signal from PBS which was quite obvious. The second group containing freshly isolated platelets also failed to contribute for any US signal generation eliminating the fact that the external membrane of platelets alone is devoid of generating any contrasts in vitro. In the third group, we found that the backfilling of the freeze-drying medium, trehalose, with $\mathrm{C}_{3} \mathrm{~F}_{8}$ also could not generate any contrast. This might be due to the fact that the trehalose could not form a stable shell to encapsulate $\mathrm{C}_{3} \mathrm{~F}_{8}$ for US imaging. In contrast to a

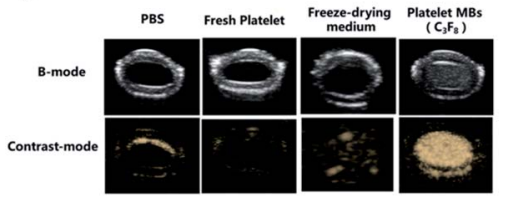

b

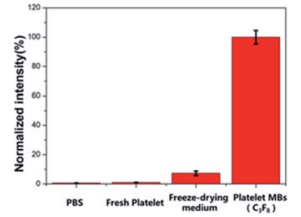

Fig. 3 (a) In vitro ultrasound contrast-enhanced images in a latex tube and (b) normalized intensity of PBS, fresh platelet, freeze-drying medium, platelet MBs.

a

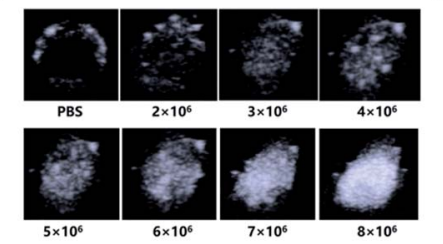

b

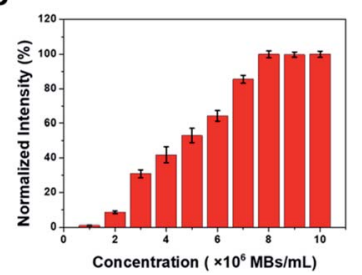

Fig. 4 (a) In vitro ultrasound contrast-enhanced images of P-UCA with increasing concentration in a latex tube. (b) Normalized US signal intensities in (a) over P-UCA concentration.

all the three groups, only the platelet MBs containing $\mathrm{C}_{3} \mathrm{~F}_{8}$ gaseous core could demonstrate significant US contrast signal (Fig. 3b). In regard to this, we further investigated the contrast enhancement with the increasing concentration of platelets in vitro.

The images collected after injecting an increasing amount of P-UCA MBs correspondingly generated increasing US signal intensities showing excellent US contrast capability. However, this was only attainable to the highest concentration of $8 \times 10^{6}$ P-UCA MBs per $\mathrm{mL}$ (Fig. 4a and b) after which the echo intensity was nearly saturated. Taken together, these results suggest that the complete membrane structure of platelet can be used as a functional carrier to encapsulate the $\mathrm{C}_{3} \mathrm{~F}_{8}$ for contrastenhanced US diagnosis. Due to inherent bio-compatibility and
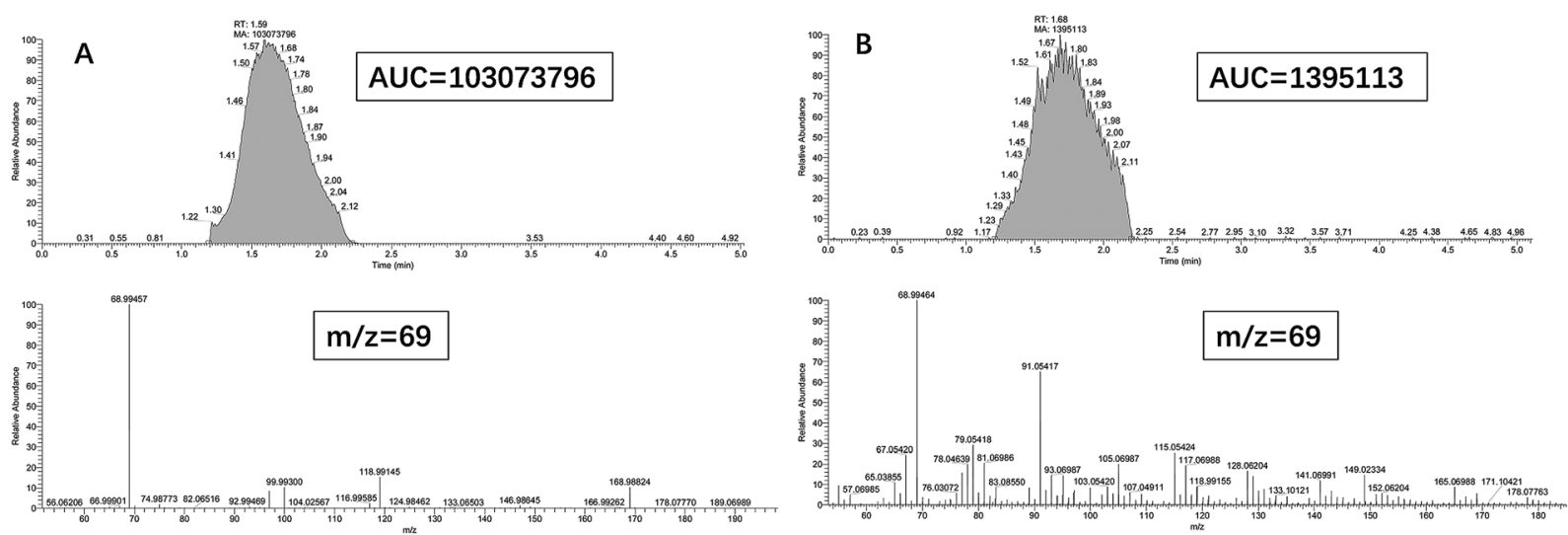

Fig. 2 GC-MS analysis of $\mathrm{C}_{3} \mathrm{~F}_{8}$ gas content in the commercial microbubble (A) and P-UCA (B). The concentration of the two contrast agents were the same $(m / z=69)$. 


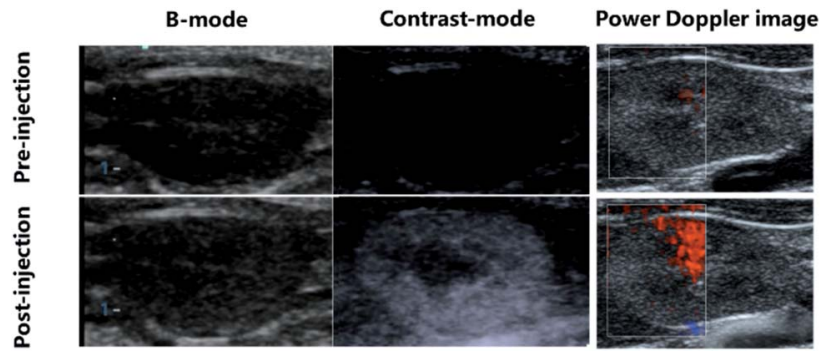

Fig. 5 In vivo ultrasound imaging of P-UCA intravenously administrated into the SD rat and the kidney was imaged with a clinical ultrasound probe (3-12 $\mathrm{MHz}$ ) by using $\mathrm{B}$-mode, contrast-mode and power Doppler-mode.

excellent biosafety, the P-UCA possess a great potential for several unique clinical application.

\subsection{In vivo ultrasound imaging}

Encouraged by the excellent in vitro ultrasound imaging capability of P-UCA, we further tested whether the P-UCA could enhance ultrasound imaging during the systemic circulation in vivo. This was verified in vivo from the contrast images of rat kidney immediately after the intravenous injection of P-UCA as shown in Fig. 5. During pre-injection of P-UCA at low MI setting (0.10), no any echo signal was apparently visible except some hyperechoic streaks which should be originally coming from the interfacial regions such as the iliopsoas surface. However, an obvious signal enhancement in the renal cortex was achieved immediately after the injection of P-UCA ultimately leading to a clear visualization of well-defined kidney margin. The details of parenchymal perfusion as observed from this technique greatly complements the gray-scale imaging ability of the platelet MBs which could be further utilized in the monitoring of several pathological conditions. Beside grayscale imaging, we further acquired the Doppler images of rat kidney in vivo similarly before and after the injection of P-UCA contrast agents. As expected, this method also showed an obvious enhancement in the Doppler signal intensity (Fig. 5)

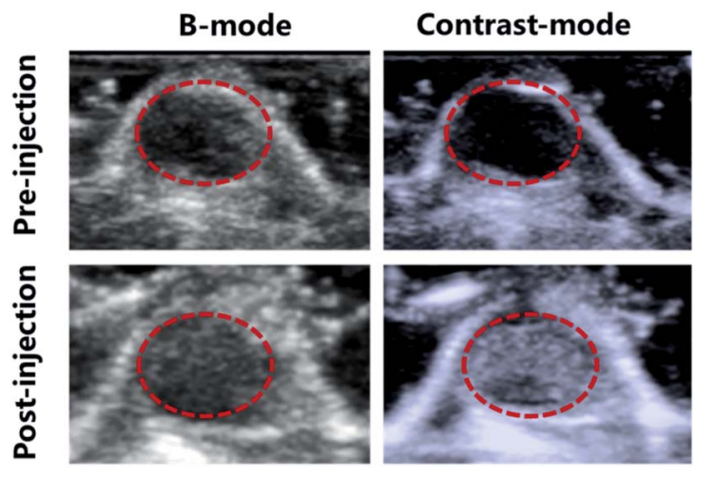

Fig. 6 P-UCA were intravenously administrated into the 4T1 tumorbearing mice and the tumor was imaged with a clinical ultrasound probe $(3-12 \mathrm{MHz})$, and the dashed circles indicate subcutaneous tumor regions. representing subtle vessels within the renal parenchyma after intravenous perfusion of the contrast agent. In brief, all the preliminary ultrasound images collected using P-UCA as a contrast agent greatly favors its further use as an optimal acoustic enhancer for both modes i.e. contrast and Doppler.

Since P-UCA MBs depicted excellent US signal generation in vivo, we further thought if these MBs could also be used as a part of clinical imaging applications such as tumor detection. To evaluate the in vivo tumor imaging ability of P-UCA, the 4T1 tumor-bearing nude mice with initial tumor volume of $\sim 100$ $\mathrm{mm}^{3}$ were intravenously injected with P-UCA via the tail vein. Initially, before injecting any dose of contrast agent we verified there was no any echo signal from the targeted tumor region, as seen in Fig. 6. After the contrast agent administration, image of the tumor was remarkably enhanced in real time, giving dynamic blood flow information with a well-defined margin of the tumor. All the above results demonstrate that P-UCA has great potential for the diagnosis of tumor and can be expanded further in monitoring other several pathological diseases both in vitro and in vivo.

In previous results, we have clearly depicted in vivo imaging ability of P-UCA including tumor imaging, however, one limitation in such imaging technique is the short imaging time. Usually, for monitoring some pathological changes and biodistribution in vivo, longer imaging time would be better. In order to overcome this limitation, we envisioned to apprehend a

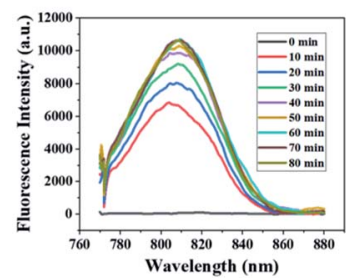

C

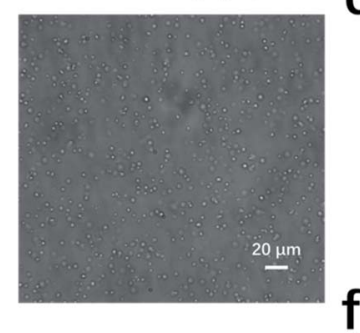

e

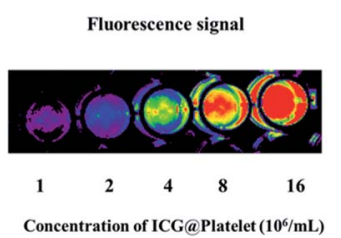

b

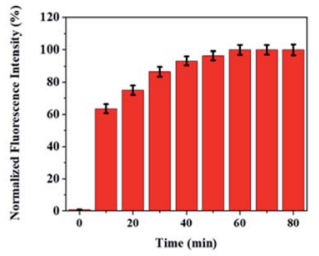

d
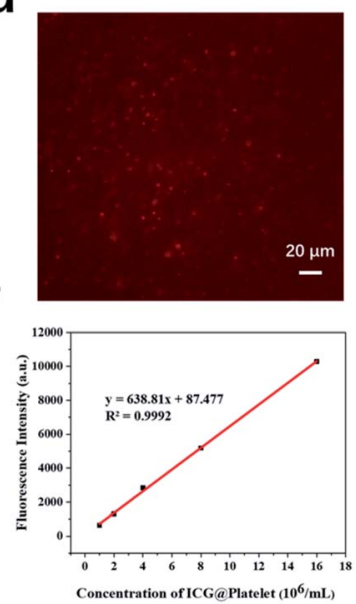

Fig. 7 (a) Fluorescence spectra and (b) normalized intensity of P-ICG after different incubation time; optical microscopy image of P-ICG under white light (c) and NIR channel (d), where red fluorescence was generated from ICG; (e) fluorescent imaging of P-ICG with different concentrations ( $f$ ) the corresponding standard curve of fluorescent signal intensities as a function of P-ICG with different concentrations. 

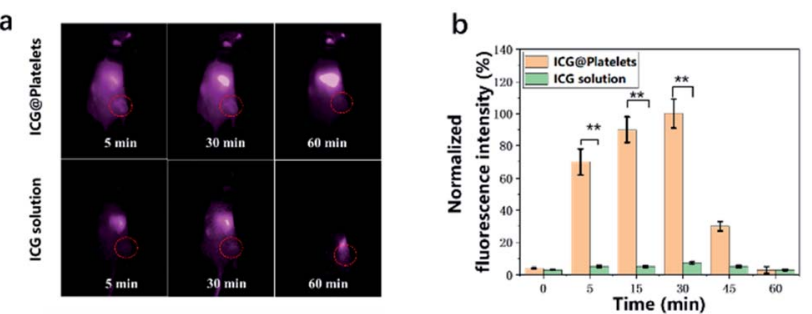

Fig. 8 (a) Fluorescence images of tumor-bearing nude mice and (b) integrated fluorescence intensity of tumor obtained after tail vein injection of P-ICG and free ICG. ${ }^{*} P<0.01$.

new imaging agent, fluorescence imaging, together with the US. Fluorescence imaging has several advantages and are wellsuited for this study. Herein, we first investigated the active loading of ICG in the platelets (P-ICG). Fig. 7a and b showed that the fluorescence intensity increased with respect to the incubation time and reached a plateau after $45 \mathrm{~min}$. This clearly illustrates that the ICG molecule can be readily encapsulated in the platelets within 45 min of incubation and the corresponding signal can be further utilized for NIRF imaging. Platelets play a vital role in a variety of biological processes, including hemostasis and wound repair. In order to balance this biological activity and fluorescence imaging capabilities of P-ICG, we choose the $45 \mathrm{~min}$ incubation time for the further preparation of P-ICG. The ICG-loaded platelets were also clearly observable under bright and NIR field (Fig. 7c and d). Next, we further quantified the ICG signal using fluorescence microscopy against different concentrations of platelets. As shown in Fig. 7e, the higher concentration of P-ICG showed significantly enhanced NIR fluorescence signal, which further supports the successful loading of ICG into platelet. A good linear relationship between the concentration of P-ICG (concentration from 1 $\times 10^{6}$ to $15 \times 10^{6}$ platelets per $\mathrm{mL}$ ) and ICG fluorescence intensity has been shown in Fig. 7f.

After a successful evaluation of fluorescence imaging in vitro, we further attempt to investigate if the P-ICG could be further used for in vivo imaging. The freshly prepared P-ICG were intravenously injected into the tumor-bearing nude mice within 1 hour. As shown in Fig. 8, despite the gradually increased fluorescence intensity in the liver, we also correspondingly found the enhanced signal in the tumor region within $5 \mathrm{~min}$ post-injection. However, no fluorescence was observed for free ICG group. Previously, we mentioned that the biological membranes can possibly eliminate limitations of ICG such as limited circulation time, photobleaching or rapid elimination from the targeted regions. Herein, the results are in close agreement that the free ICG molecules have been already washed out while P-ICG could still show longer tumor retention time in vivo. This can be mainly because platelet itself belong to the body, thereby shielding ICG from the immune system. Importantly, we could observe the increase in time of fluorescence imaging for the platelet-based delivery system, although not sufficiently large but to the extent required for the intraoperative imaging.

\section{Conclusions}

In summary, we have successfully constructed $\mathrm{C}_{3} \mathrm{~F}_{8}$ encapsulated platelet (P-UCA) and ICG loaded platelet (P-ICG) for a realtime ultrasound and fluorescence imaging especially for monitoring the pathological process of platelets both in vitro and in vivo. The multisizer measurements showed that more than $80 \%$ of the platelets could be recovered from the freezedried powder with a mean particle diameter of $3.1 \pm 0.4 \mu \mathrm{m}$ concluding that these sizes ranged within an appropriate limit as required for the intravenous administration. The as-prepared P-UCA maintained the integrity of the platelet structure and successfully encapsulated $\mathrm{C}_{3} \mathrm{~F}_{8}$ gas, thus endowed platelet with significant ultrasound contrast-enhancing ability in vitro and in vivo. Besides, we further demonstrated that these platelets could facilitate active loading of ICG molecules which was sufficient for prolonged in vivo fluorescence imaging compared with free ICG to monitor the bio-distribution. During the process, the tumor boundary could be observed clearly for more than $30 \mathrm{~min}$. This is a proof-of-concept study that depicts the first-hand synthesis and evaluation of whole platelets MBs for direct real-time imaging in two different modalities i.e. ultrasound and fluorescence imaging. Herein, we have established a new vision to direct future imaging research based on naive membrane-based materials that have intact surface and retain all the necessary functional moieties that would ultimately direct its inherent biological function in vivo for long-term tracking applications especially in the progression of various pathological diseases.

\section{Experimental section}

\subsection{Materials}

4-(2-Hydroxyethyl)-1-piperazineethanesulfonic acid (HEPES), prostaglandin-E1 (PGE1), ethylenebis(oxyethylenenitrilo)tetraacetic acid (EGTA), imidazol, trehalose, indocyanine green (ICG) were purchased from TCI (Shanghai, China). All other reagents and solvents were purchased from the domestic suppliers and used as received.

\subsection{Isolation of platelets}

Murine platelets were isolated as described previously. In brief, whole blood was collected from the SD rat or BALB/c mice into a heparin-treated blood collection tube and centrifuged at $100 \mathrm{~g}$ for $20 \mathrm{~min}$ at $25^{\circ} \mathrm{C}$. The platelet-rich plasma (PRP) was transferred to a separate tube using a transfer pipette (wide orifice), and prostaglandin-E1 was added to each tube at a final concentration of $1 \mu \mathrm{M}$.

Platelets were isolated from the PRP via centrifugation at $800 \mathrm{~g}$ for $10 \mathrm{~min}$. The plasma was discarded, and the platelets were resuspended carefully and slowly in buffer A ( $\mathrm{pH}$ 6.8, $10 \mathrm{mM} \mathrm{KCl}, 100 \mathrm{mM} \mathrm{NaCl}, 10 \mathrm{mM}$ imidazole, $10 \mathrm{mM}$ EGTA, 10 $\mu \mathrm{g} \mathrm{mL}^{-1}$ prostaglandin-E1). Note that this buffer was released slowly along the tube wall while minimizing agitation. Platelets were counted using a cell counter (Multisizer 4), washed platelets at a concentration of $1-2 \times 10^{9}$ platelets per $\mathrm{mL}$ were 
incubated at $37^{\circ} \mathrm{C}$ for $4 \mathrm{~h}$ in buffer $\mathrm{A}$ in the presence of $45 \mathrm{mM}$ trehalose. After incubation, platelets were pelleted $(25 \mathrm{~s}$ in an Eppendorf $5415 \mathrm{C}$ microfuge) and resuspended in lyophilization buffer (9.5 mM HEPES, $142.5 \mathrm{mM} \mathrm{NaCl}, 4.8 \mathrm{mM} \mathrm{KCl,} 1 \mathrm{mM}$ $\mathrm{MgCl}_{2}, 30 \mathrm{mM}$ trehalose).

\subsection{Freeze-drying}

The platelets were further lyophilized via freeze-drying experiments using a mid-scale research-level lyophilizer (Virtis, ADVANTAGE EL-85). A siliconized glass vial of total volume capacity $3.7 \mathrm{~mL}$ was chosen to transfer $1 \mathrm{~mL}$ platelet solution. The vials were then placed on the lyophilizer shelves and frozen at $-60{ }^{\circ} \mathrm{C}$. The sample cooling rate of $2{ }^{\circ} \mathrm{C} \min ^{-1}$ was set starting from the initial $22^{\circ} \mathrm{C}$. The sample was left for approximately $2 \mathrm{~h}$ at $-60{ }^{\circ} \mathrm{C}$ and subsequently, pressure inside the chamber was then reduced to 100 mTorr. After reduced pressure, the shelves were further heated from $-60{ }^{\circ} \mathrm{C}$ to $-30{ }^{\circ} \mathrm{C}$ at a rate of $0.75{ }^{\circ} \mathrm{C} \min ^{-1}$ and the samples were left for approximately $4 \mathrm{~h}$ at that temperature.

Once the sample reached $-30{ }^{\circ} \mathrm{C}$ for $4 \mathrm{~h}$, the shelves temperature were further increased to $20{ }^{\circ} \mathrm{C}$ at a rate of $0.2{ }^{\circ} \mathrm{C} \mathrm{min}^{-1}$. The samples were left overnight under vacuum at this temperature. The freeze-dried samples were then perfused with $\mathrm{C}_{3} \mathrm{~F}_{8}$ gas and stored at room temperature after proper sealing with rubber stoppers. The lyophilized sample measured $2 \pm 1 \%$ water content on a dry weight basis (wt/wt\%).

\subsection{Gas chromatograph-mass spectrometer (GC-MS)}

The GC-MS is carried out on Q Exactive GC (Thermofisher). Gas chromatographic conditions are as follows: the column is HPVOC $(30 \mathrm{~m} \times 0.32 \mathrm{~mm} \times 1.8 \mu \mathrm{m})$; the carrier gas is helium; the column flow rate is $1.2 \mathrm{~mL} \mathrm{~min}^{-1}$; the column temperature program: the initial temperature is $40{ }^{\circ} \mathrm{C}$ and keeps for $5 \mathrm{~min}$, then the temperature rises at the rate of $15{ }^{\circ} \mathrm{C} \mathrm{min}^{-1}$ until the temperature raised to $230{ }^{\circ} \mathrm{C}$ and keeps for $5 \mathrm{~min}$. Mass spectrometry conditions are as follows: EI ionization source, doubling voltage $1600 \mathrm{~V}$; selected ions: $\mathrm{m} / \mathrm{z}$ (mass ratio) 69, 169 . The operation steps are as follows: $3 \mathrm{~mL}$ of the prepared microbubble suspension (or $100 \mu \mathrm{L} \mathrm{C}_{3} \mathrm{~F}_{8}$, corresponding to the gas content in the commercial microbubble) is accurately aspirated, then injected into a gas bag containing $500 \mathrm{~mL}$ highpurity nitrogen, and incubated for 24 hours in a water bath (37 ${ }^{\circ} \mathrm{C}$ ) to completely rupture the microbubbles to release $\mathrm{C}_{3} \mathrm{~F}_{8}$ gas. $50 \mu \mathrm{L}$ of the above gas is subjected to analysis, with high purity nitrogen as a control.

\subsection{Characterization of P-UCA}

The optical characterization of as prepared MBs was mostly carried out using a Leica DM 4500P microscope attached to a Leica DFC 420 digital camera. Further, Coulter multisizer III (Coulter Electronics Ltd., Luton, Bedfordshire, UK) was used to measure the size distribution and also the concentration of the P-UCA MBs. For in vivo acoustic test, the sample was normalized to a final concentration of $1.0 \times 10^{8}$ bubbles per $\mathrm{mL}$ (using PBS). In most of the times, prior to the use of MBs, the vials containing MBs were gently rotated to ensure that these MBs were distributed evenly.

\subsection{Ultrasound imaging}

The platelet MBs were prepared for in vitro ultrasonography using a latex tube of inner diameter approx. $5 \mathrm{~mm}$. A broadband linear array L9-3 transducer (7 to $3 \mathrm{MHz}$ extended) of IU22 ultrasound system (Philips Medical Systems) was chosen for recording all the images. During the imaging, different concentration of platelet MBs was prepared after dispersing well in the PBS solution and injected to the latex tube. All the images were acquired from the tube cross-section in both PIHI (phase-inversion harmonic imaging) and conventional B-mode.

Similarly, for the in vivo study, SD rat $(n=3)$ of average weight $280 \mathrm{~g}$ were subjected to anesthesia by intraperitoneal injection of pentobarbital sodium. The animals were left on a warm blanket until the body temperature raised within normal range. For in vivo imaging studies, $200 \mu \mathrm{L}$ of platelet MBs at the concentration of $1 \times 10^{8}$ platelets per $\mathrm{mL}$ dissolved in PBS were injected via a catheter and later flushed with $300 \mu \mathrm{L}$ of saline. The MBs injected rats were then subjected for kidney imaging using a broadband L9-3 transducer in both conventional B-mode and PIHI mode. The recorded images were saved in an offline directory for future off-line review. A similar technique was followed to image the tumor tissues as well. All the animal experiments were approved by the Institutional Animal Care and Use Committee of Peking University and carried out ethically and humanely.

\subsection{ICG loading}

Platelets were isolated from the PRP via centrifugation at $800 \mathrm{~g}$ for $10 \mathrm{~min}$. The plasma was discarded, and the platelets were resuspended carefully and slowly in $10 \mu \mathrm{g} \mathrm{mL}^{-1}$ ICG solution (sucrose isotonic solution containing $1 \mu \mathrm{M}$ PGE1). The mixture was gently shaken and incubated at $37^{\circ} \mathrm{C}$ for $0,10,20,30,40$, $50,60,70,80 \mathrm{~min}$ at $100 \mathrm{rpm}$ in the dark. Then, the platelets were isolated from the mixture via centrifugation at $800 \mathrm{~g}$ for $10 \mathrm{~min}$. The supernatant was discarded and the platelets were resuspended carefully and slowly in PBS and washed for twice. Fluorescence spectra were performed to study the fluorescence imaging ability of ICG loaded platelets. Fluorescence microscopy was performed to determine the encapsulated ICG in platelets. One drop of the ICG-platelet suspension was placed on a clean glass slide. The glass slide was observed under a fluorescence microscope.

\subsection{Fluorescence imaging}

NIR fluorescence imaging experiments were performed and analyzed using the Fluorescence-labeled Organism Bioimaging Instrument (FOBI, Korea) under certain parameters $\left(\lambda_{\mathrm{ex}}=\right.$ $740 \mathrm{~nm}, \lambda_{\mathrm{em}}=750-800 \mathrm{~nm}$, exposure time $\left.=0.1 \mathrm{~s}\right)$. Tumorbearing mice were i.v. injected with $100 \mu \mathrm{L}$ of ICG-platelet (1 $\mathrm{mg} \mathrm{mL} \mathrm{mL}^{-1}$ ). NIR fluorescence images of the mice were acquired at different time points after injection. 


\subsection{Statistical analysis}

Data were presented as mean \pm standard deviation (SD). Student's $t$-test was applied to test the significance of the difference, which was considered to be significant when $p<$ 0.05 .

\section{Ethics statement}

In the present study, all experiments were performed under protocols approved by the Institutional Animal Care and Use Committee (IACUC) of Peking University and were in strict accordance with the principles and guidelines of the National Institutes of Health Guide for the Care and Use of Laboratory Animals. The Animal Care and Use Committees of Peking University approved all animal care and experimental protocols.

\section{Conflicts of interest}

The authors declare no competing financial interest.

\section{Acknowledgements}

National Key Research and Development Program of China (No. 2016YFA0201400), Beijing Natural Science Foundation - Haidian original innovation joint fund (No. 17L20170) and the Foundation for Innovative Research Groups of the National Natural Science Foundation of China (No. 81421004).

\section{References}

1 C. Jenne, R. Urrutia and P. Kubes, Platelets: bridging hemostasis, inflammation, and immunity, International Journal of Laboratory Hematology, 2013, 35(3), 254-261.

2 M. T. Rondina, A. S. Weyrich and G. A. Zimmerman, Platelets as cellular effectors of inflammation in vascular diseases, Circ. Res., 2013, 112(11), 1506-1519.

3 A. Moers, B. Nieswandt, S. Massberg, N. Wettschureck, S. Grüner, I. Konrad, et al., G13 is an essential mediator of platelet activation in hemostasis and thrombosis, Nat. Med., 2003, 9(11), 1418.

4 V. Plaks, C. D. Koopman and Z. Werb, Circulating tumor cells, Science, 2013, 341(6151), 1186-1188.
5 L. J. Gay and B. Felding-Habermann, Contribution of platelets to tumour metastasis, Nat. Rev. Cancer, 2011, 11(2), 123.

6 L. Borsig, The role of platelet activation in tumor metastasis, Expert Rev. Anticancer Ther., 2008, 8(8), 1247-1255.

7 W. S. Nesbitt, E. Westein, F. J. Tovar-Lopez, E. Tolouei, A. Mitchell, J. Fu, et al., A shear gradient-dependent platelet aggregation mechanism drives thrombus formation, Nat. Med., 2009, 15(6), 665.

8 S. Nandi and A. C. Brown, Platelet-mimetic strategies for modulating the wound environment and inflammatory responses, Exp. Biol. Med., 2016, 241(10), 1138-1148.

9 G. Hong, A. L. Antaris and H. Dai, Near-infrared fluorophores for biomedical imaging, Nat. Biomed. Eng., 2017, 1(1), 0010.

10 L. Dai, N. Gu, B.-A. Chen and G. Marriott, Human platelets repurposed as vehicles for in vivo imaging of myeloma xenotransplants, Oncotarget, 2016, 7(16), 21076.

11 J.-M. I. Maarek, D. P. Holschneider and J. Harimoto, Fluorescence of indocyanine green in blood: intensity dependence on concentration and stabilization with sodium polyaspartate, J. Photochem. Photobiol., B, 2001, 65(2-3), 157-164.

12 T. Desmettre, J. Devoisselle and S. Mordon, Fluorescence properties and metabolic features of indocyanine green (ICG) as related to angiography, Surv. Ophthalmol., 2000, 45(1), 15-27.

$13 \mathrm{~J}$. Yu, M. A. Yaseen, B. Anvari and M. S. Wong, Synthesis of near-infrared-absorbing nanoparticle-assembled capsules, Chem. Mater., 2007, 19(6), 1277-1284.

14 R. Manchanda, A. Fernandez-Fernandez, A. Nagesetti and A. J. McGoron, Preparation and characterization of a polymeric (PLGA) nanoparticulate drug delivery system with simultaneous incorporation of chemotherapeutic and thermo-optical agents, Colloids Surf., B, 2010, 75(1), 260-267.

15 M. Rudin, Noninvasive structural, functional, and molecular imaging in drug development, Curr. Opin. Chem. Biol., 2009, 13(3), 360-371.

16 J. Yan, M. C. Estévez, J. E. Smith, K. Wang, X. He, L. Wang and W. Tan, Dye-doped nanoparticles for bioanalysis, Nano today, 2007, 2(3), 44-50. 